\title{
THE NUTATIONS OF NEUTRON STARS AND CORE-CRUST COUPLING
}

\author{
C. R. Gwinn \\ Harvard-Smithsonian Center for Astrophysics
}

Neutron stars, like the earth, are rotating fluid-filled ellipsoids. Poincaré (Bull. Astron. 27, 321, 1910), Hough (Phil. Trans. R. Soc. A186, 469, 1895) and others have discussed the nutations of such objects through a simple model, which treats the crust as rigid and the core as an ideal fluid of uniform density and vorticity. The core and crust are coupled by inertial coupling: the forces which constrain the fluid to its cavity within the crust can produce a net torque, since the cavity is ellipsoidal. Additional torques, and the effects of the elasticity in the crust and density stratification in the core, may be accomodated in such models as well (Sasao et al., Proc. IAU Symposium 78, p. 165, 1980, and references therein).

Such a system has two nutational normal modes, corresponding to the earth's Chandler Wobble (CW) and free core nutation (FCN). The frequencies of the modes in an inertial reference frame are:

$$
n_{C W}=\Omega\left(1-\frac{A}{A_{s}} e\right)
$$

and

$$
n_{F C N}=-\Omega \frac{A}{A_{0}}\left[e_{f}-\imath \frac{\kappa}{A_{f}}\right] .
$$

Here, $\Omega$ is the star's rotation rate. The equatorial moment of inertia of the entire star is $A$, and the polar moment of inertia is $C$. The dynamical ellipticity of the star is $e=\frac{C-A}{A}$. Analogous quantities $A_{f}, e_{f}$ refer to the core, and $A_{0}$ to the crust. Possible perturbations to the inertial coupling are parametrized by $\kappa$.

These modes produce very different motions (Toomre, Geophys. J. R. Astron. Soc. 38, 335, 1974). In particular, only the FCN involves large relative motion between core and crust, and only it is sensitive to core-crust coupling. Other than inertial coupling, core-crust coupling via the core plasma may be important (Sauls et al., Phys. Rev. D 25, 967, 1982; Alpar et al., Ap. J. 282, 533, 1984), particularly in determining the $Q$ of the FCN. The superfluidity of the core fluid in neutron stars should not affect the validity of the model. Shaham (Ap. J. 214, 251, 1977) has pointed out that pinning of superfluid vortices to crust nuclei may increase the crust's effective moment of inertia; core-crust coupling by vortex pinning is orders of magnitude less than the inertial coupling torque. Observations of the frequency and $Q$ of free nutations of neutron stars, and identification of the mode responsible, should usefully constrain neutron star models. 